\title{
Tests and applications of nonlinear force-free field extrapolations in spherical geometry
}

\author{
Y. Guo ${ }^{1,2}$ and M. D. Ding ${ }^{1,2}$ \\ ${ }^{1}$ School of Astronomy and Space Science, Nanjing University, Nanjing 210093, China \\ email: guoyang@nju.edu.cn \\ ${ }^{2}$ Key Laboratory of Modern Astronomy and Astrophysics (Nanjing University), Ministry of \\ Education, Nanjing 210093, China
}

\begin{abstract}
We test a nonlinear force-free field (NLFFF) optimization code in spherical geometry with an analytical solution from Low and Lou. The potential field source surface (PFSS) model is served as the initial and boundary conditions where observed data are not available. The analytical solution can be well recovered if the boundary and initial conditions are properly handled. Next, we discuss the preprocessing procedure for the noisy bottom boundary data, and find that preprocessing is necessary for NLFFF extrapolations when we use the observed photospheric magnetic field as bottom boundaries. Finally, we apply the NLFFF model to a solar area where four active regions interacting with each other. An M8.7 flare occurred in one active region. NLFFF modeling in spherical geometry simultaneously constructs the small and large scale magnetic field configurations better than the PFSS model does.
\end{abstract}

Keywords. Sun: activity, Sun: corona, Sun: magnetic fields

Nonlinear force-free field (NLFFF) extrapolations provide more information for the solar magnetic field, such as free magnetic energy and highly sheared magnetic field lines, compared to potential magnetic field models. In order to study large scale magnetic field connections between different active regions, the curvature of the solar surface cannot be ignored, which asks for magnetic field modeling in spherical geometry. However, there are only a few NLFFF procedures in spherical geometry, such as the optimization method proposed by Wheatland et al. (2000) and implemented by Wiegelmann (2007) and the magnetohydrodynamics (MHD) relaxation method implemented by van Ballegooijen (2004) and by Jiang et al. (2012) independenly. Another version of the optimization method in spherical geometry has been implemented by J. McTiernan in the FORTRAN language and released in the Solar Software (SSW). Although Tadesse et al. (2009) have tested the optimization code in spherical geometry implemented by Wiegelmann (2007), it is still necessary to test J. McTiernan's version of the code before we apply it to observations.

We adopt an analytical NLFFF solution from Low \& Lou (1990) as the benchmark for testing J. McTiernan's version of the optimization code. The potential field source surface (PFSS; Schrijver \& DeRosa 2003) model is used as the initial condition. The computation domain is a wedge-shaped volume that is bounded by six boundaries with $r \in\left[r_{\min }, r_{\max }\right], \theta \in\left[\theta_{\min }, \theta_{\max }\right]$, and $\phi \in\left[\phi_{\min }, \phi_{\max }\right]$. For boundary conditions in a test case with the Low and Lou solution, all the six boundaries can be specified. In such a case, the analytical solution can be well recovered to the numerical accuracy. To simulate realistic observations, only the bottom boundary condition is specified by the analytical vector fields. The boundary conditions on the other five surfaces are specified by the PFSS model. In such a case, the analytical solution cannot be well recovered. This is because the lateral and top boundary conditions, which are provided by the PFSS model, do 
not match with the NLFFF model. To reduce the boundary effect, we use a weighting function in a buffer zone with six grid points toward the top and lateral boundaries. The weighting function decreases from 1 to 0 from inside to outside with a cosine profile. Test results show that a weighting function improves the NLFFF extrapolation.

Molodensky (1969) and Aly (1989) pointed out that the magnetic force-free and torquefree conditions must be satisfied for vector magnetic fields on a closed boundary of any force-free volume. For practical observations, only magnetic fields on the bottom boundary are available, and they are usually not force-free. Therefore, the observed boundary data should be preprocessed in an isolated region to remove the net magnetic force and magnetic torque (Wiegelmann et al. 2006). The preprocessing routine in spherical geometry has been developed by Tadesse et al. (2009). We have written another version of the preprocessing code following Tadesse et al. (2009). In order to test the preprocessing code, we prepare a noisy bottom boundary by adding Gaussian-distributed random noises to the bottom surface of the Low and Lou solution. Then, two NLFFF extrapolations are conducted with different bottom conditions, one with the preprocessed data and the other with the noisy data. Comparisons of the two extrapolations show that the preprocessing procedure improves the extrapolation results.

We apply the NLFFF model in spherical geometry to the vector magnetic fields observed by the Helioseismic and Magnetic Imager (HMI; Scherrer et al. 2012) on board the Solar Dynamic Observatory (SDO). The region of interest includes four active regions, in one of which an M8.7 class flare occurred. The magnetic field configurations both before and after the flare are constructed. The extreme ultraviolet (EUV) coronal loops observed by the Atmospheric Imaging Assembly (AIA; Lemen et al. 2012) on board SDO show a highly non-potential structure in the flare-productive region before the flare. The projection of NLFFF field lines matches both the lower filament structure and the higher EUV coronal loops better than the PFSS model does. After the flare, the two models have less differences from each other. A more detailed analysis on the aforementioned studies can be found in Guo et al. (2012).

\section{References}

Aly, J. J. 1989, Solar Phys., 120, 19

Guo, Y., Ding, M. D., Liu, Y., Sun, X. D., DeRosa, M. L., \& Wiegelmann, T. 2012, Astrophys. $J ., 760,47$

Jiang, C., Feng, X., \& Xiang, C. 2012, Astrophys. J., 755, 62

Lemen, J. R., Title, A. M., Akin, D. J., Boerner, P. F., Chou, C., Drake, J. F., et al. 2012, Solar Phys., 275, 17

Low, B. C. \& Lou, Y. Q. 1990, Astrophys. J., 352, 343

Molodensky, M. M. 1969, Sov. Astronom., 12, 585

Scherrer, P. H., Schou, J., Bush, R. I., Kosovichev, A. G., Bogart, R. S., Hoeksema, J. T., et al. 2012, Solar Phys., 275, 207

Schrijver, C. J. \& DeRosa, M. L. 2003, Solar Phys., 212, 165

Tadesse, T., Wiegelmann, T., \& Inhester, B. 2009, Astron. Astrophys., 508, 421

van Ballegooijen, A. A. 2004, Astrophys. J., 612, 519

Wheatland, M. S., Sturrock, P. A., \& Roumeliotis, G. 2000, Astrophys. J., 540, 1150

Wiegelmann, T. 2007, Solar Phys., 240, 227

Wiegelmann, T., Inhester, B., \& Sakurai, T. 2006, Solar Phys., 233, 215 